\title{
Benefícios para a gestante com a participação paterna no pré-natal: uma revisão integrativa
}

Benefits for pregnant women with paternal participation in prenatal care: an integrative

Beneficios para mujeres embarazadas con participación paterna en la atención prenatal: una revisión integradora

\section{RESUMO}

Objetivo: identificar os benefícios propiciados à gestante em decorrência da participação paterna durante o pré-natal. Método: Trata-se de uma revisão integrativa da literatura realizada a partir das bases de dados SciELO, LILACS, BDENF e MEDLINE entre os meses de setembro e outubro de 2020. Para definição da pergunta norteadora utilizou-se a estratégia PICO. As buscas abrangeram o período de 2014 a 2020. Resultados: a participação paterna durante o pré-natal é de suma importância para favorecer maior vínculo entre o casal e proporcionar um ambiente acolhedor para o recém-nascido. Além disso, o suporte paterno durante o pré-natal é capaz de trazer sentimentos positivos para a mulher e isso influencia no decorrer da gestação. Conclusão: Desafios precisam ser superados para maior participação dos homens no acompanhamento pré-natal como dos horários das consultas e coincidir com o horário de trabalho. Conclui-se, portanto, que a participação do pai gera um vínculo muito importante tanto na vida da criança como na da gestante.

DESCRITORES: Saúde do homem; Paternidade; Cuidado pré-natal; Gravidez.

\section{ABSTRACT}

Objective: To identify the benefits provided to pregnant women as a result of paternal participation during prenatal care. Method: This is an integrative literature review carried out from the SciELO, LILACS, BDENF and MEDLINE databases between the months of September and October 2020. The PICO strategy was used to define the guiding question. The searches covered the period from 2014 to 2020. Results: Paternal participation during prenatal care is of paramount importance to favor a greater bond between the couple and provide a welcoming environment for the newborn. In addition, paternal support during prenatal care is able to bring positive feelings to the woman and this influences the course of pregnancy. Conclusion: Challenges need to be overcome for greater participation of men in prenatal care, such as appointment times and coincide with work hours. It is concluded, therefore, that the participation of the father generates a very important bond both in the life of the child and that of the pregnant woman.

DESCRIPTORS: Men's health; Paternity; Prenatal care; Pregnancy.

\section{RESUMEN}

Objetivo: Identificar los beneficios que se brindan a las gestantes como resultado de la participación paterna durante la atención prenatal. Método: Se trata de una revisión integradora de la literatura realizada a partir de las bases de datos SciELO, LILACS, BDENF y MEDLINE entre los meses de septiembre y octubre de 2020. Se utilizó la estrategia PICO para definir la pregunta orientadora. Las búsquedas abarcaron el período de 2014 a 2020. Resultados: La participación de los padres durante la atención prenatal es de suma importancia para favorecer un mayor vínculo entre la pareja y brindar un ambiente acogedor para el recién nacido. Además, el apoyo paterno durante el cuidado prenatal puede traer sentimientos positivos a la mujer y esto influye en el curso del embarazo. Conclusión: Es necesario superar desafíos para una mayor participación de los hombres en la atención prenatal, como los horarios de las citas y la coincidencia con el horario laboral. Se concluye, por tanto, que la participación del padre genera un vínculo muy importante tanto en la vida del niño como en la de la embarazada.

DESCRIPTORES: Salud del hombre; Paternidad; Atención prenatal; Embarazo.

RECEBIDO EM: 04/04/2021 APROVADO EM: 26/04/2021

\section{Ariele Ferreira Vieira}

Curso de Graduação em Enfermagem. Universidade Paulista (UNIP), Campus Brasília-DF.

ORCID: 0000-0001-9910-6507 


\section{Ricardo Saraiva Aguiar}

Professor Assistente. Curso de Graduação em Enfermagem. Universidade Paulista (UNIP), Campus Brasília-DF. ORCID: 0000-0003-0335-2194

\section{INTRODUÇÃO}

A gestação ocasiona transformações no corpo, na mente e no cotidiano das mulheres. É o momento de buscar atendimento especializado para o início precoce do pré-natal de modo a preparar a família para o parto e acompanhar as demandas de saúde desta mulher e de sua família para que a gravidez transcorra de forma tranquila. Nessa fase, é fundamental que a gestante conte com o pleno apoio familiar, em especial do pai da criança. ${ }^{1}$

Diante disso, o Ministério da Saúde instituiu de forma oficial na Política Nacional de Atenção Integral à Saúde (PNAISH) o incentivo à participação paterna no pré-natal para preparar e tornar o homem agente de transformação social e familiar no cuidado da mulher e de sua própria saúde. ${ }^{2-3}$ A PNAISH tem por objetivo promover a procura e ampliar o acesso da população masculina aos serviços de saúde, sendo a primeira política de saúde da América Latina voltada exclusivamente aos homens. ${ }^{4-5}$

A PNAISH visa implantar e/ou estimular nos serviços de saúde, públicos e privados, uma rede de atenção integral à saúde do homem que garanta linhas de cuidado na perspectiva da integralidade capazes de formar e qualificar os profissionais para o seu adequado atendimento. ${ }^{6}$

Nessa perspectiva, a interação do homem nas etapas da gravidez vem aumentando em função da responsabilidade em promover o efetivo comprometimento com a paternidade consciente e mudanças sexuais impostas pelas conversões nos papéis dos genitores. Diante disso, torna-se fundamental a participação dos profissionais de saúde na promoção dos sentimentos de competência e de confiança ao genitor, reforçando sua valiosa contribuição para a saúde de seu filho. ${ }^{6-7}$

A atenção primária à saúde (APS) deve ser a porta de entrada dos usuários no sistema de serviços de saúde de forma a satisfazer as principais necessidades por meio de um conjunto de ações individuais e coletivas que envolvem a promoção, a prevenção, o diagnóstico, o tratamento e a reabilitação. ${ }^{8-9}$

Os estudos já realizados sobre a presença do pai na gestação, parto e puerpério apontam que este pode apoiar a companheira de diversas formas. Pode acompanhá-la nas consultas e exames; elogiar, mostrando sua percepção, as modificações no seu corpo; conversar mais com ela, sendo compreensivo e prestativo; ajudá-la com as tarefas domésticas, principalmente se ela trabalhar fora ou se o casal já tiver outros filhos. $\mathrm{O}$ contato do pai com os exames, como as ecografias, ajuda na materialização da presença do novo filho, iniciando o vínculo emocional ainda na gestação. ${ }^{10-12}$

Nesse contexto, o presente estudo tem o objetivo de identificar os benefícios propiciados à gestante em decorrência da participação paterna durante o pré-natal. Sob esta perspectiva, destaca-se a questão que norteou a presente proposta investigativa: Quais os benefícios gestacionais propiciados pela inclusão paterna no pré-natal?

\section{MÉTODOS}

Trata-se de uma revisão integrativa da literatura realizada em sete etapas13: 1) delimitação da pergunta norteadora da revisão; 2) definição dos critérios de inclusão e exclusão; 3 ) busca extensiva da literatura; 4) identificação de potenciais estudos por meio de avaliação do título e resumo; 5) seleção dos artigos com base no texto completo; 6) avaliação da qualidade dos estudos inclusos; 7) síntese dos estudos inclusos.

Tendo em vista a primeira fase da revisão, elaborou-se a pergunta norteadora de pesquisa com base na estratégia PICO: P - população e problema; I - intervenção; C - comparação; e O - outcome (termo em inglês que significa desfecho).14 Assim, considerou-se P: gestantes; I: benefícios gestacionais; C: qualquer comparação sobre a participação paterna; $O$ : assistência ao pré-natal. Nesta direção, a pergunta construída foi: Quais os benefícios gestacionais propiciados pela inclusão paterna no pré-natal?

A busca dos artigos foi realizada entre os meses de setembro e outubro de 2020 nas bases de dados eletrônicas Scientific Electronic Library Online (SciELO), Literatura Científica e Técnica da América Latina e Caribe (LILACS), Base de Dados em Enfermagem (BDENF) e Medical Literature Analysis and Retrievel System Online (Medline).

Para definição dos termos de busca, foi realizada consulta aos Descritores em Ciências da Saúde (DeCS). Elegeu-se o descritor "saúde do homem" que foi combinado com o termo de busca "paternidade", "cuidado pré-natal" e "gravidez". Utilizou-se os operadores booleano "AND" e "OR" para combinação. As estratégias construídas com os termos de busca e seus resultados são apresentados no Quadro 1.

Quadro 1. Estratégias de busca e resultados das produções identificadas. Brasilia, Distrito Federal, 2020.

\begin{tabular}{|c|c|c|}
\hline FONTES DE INFORMAÇÃO & EXPRESSÕES DE BUSCA & RESULTADOS \\
\hline SCIELO & "saúde do homem" OR "paternidade" AND "cuidado pré-natal" OR "gravidez" & 32 \\
\hline LILACS & "saúde do homem" OR "paternidade" AND "cuidado pré-natal" OR "gravidez" & 131 \\
\hline BDENF & "saúde do homem" AND "paternidade" AND "cuidado pré-natal" OR "gravidez" & 5 \\
\hline
\end{tabular}




\section{artigo}

Vieira, A.F.; Aguiar, R.S.

Benefícios para a gestante com a participação paterna no pré-natal: uma revisão integrativa

\begin{tabular}{|l|c|c|}
\hline \multicolumn{1}{|c|}{ MEDLINE } & '“Men's Health" AND “Paternity" AND "Prenatal Care” & 24 \\
\hline & TOTAL & 192 \\
\hline Elaboração: Vieira AF, Aguiar RS, 2020. & \\
\hline
\end{tabular}

Teve-se como critérios de inclusão para a amostra: artigos publicados de forma online nos últimos 6 anos (2014 a 2020); disponíveis em língua portuguesa e na íntegra; estudos no formato de artigos originais oriundos de produções científicas diversificadas. Como critérios de exclusão, enquadraram-se comentários, reflexões, dissertações e teses.

A busca nas bases de dados gerou 192 referências. Dessas, 32 estavam no SciELO, 131 na LILACS, 24 na MEDLINE e 5 artigos na BDENF. Reduziu-se, a partir da aplicação dos filtros de inclusão, o número de ocorrência: 24 artigos por estarem duplicados, 34 artigos por terem tema diverso do objetivo proposto e 20 artigos devido a metodologia. Totalizaram-se 114 artigos submetidos à leitura completa e à aplicação dos critérios de exclusão, gerando-se a rejeição de 63 artigos. Após a leitura completa dos artigos foram rejeitados ainda 41 artigos por não responderem à questão de pesquisa. Constituiu-se assim a amostra revisada de 10 artigos (Figura 1).

Classificaram-se as evidências dos artigos em seis níveis: Nível I - estudos relacionados à metanálise de múltiplos estudos controlados; Nível II - estudos experimentais individuais; Nível III - estudos quase-experimentais, como o ensaio clínico não randomizado, o grupo único pré e pós-teste, além de séries temporais ou caso-controle; Nível IV - estudos não experimentais, como a pesquisa descritiva, correlacional e comparativa, com abordagem qualitativa e estudos de caso; Nível V - dados de avaliação de programas obtidos de forma sistemática; e Nível VI - opiniões de especialistas, relatos de experiência, consensos, regulamentos e legislações. ${ }^{15}$

Os dados compilados foram então analisados por meio da análise temática16, sendo organizados e apresentados em categorias temáticas obtidas a partir das seguintes etapas de análise: 1) familiarização dos dados (resultados dos estudos que compuseram a amostra e se relacionavam com a pergunta da pesquisa); 2) geração de códigos iniciais; 3 ) busca por temas; 4) revisão dos temas; 5) definição e titulação dos temas; 6) produção do relatório.

\section{RESULTADOS}

Elaborou-se, para facilitar a extração e síntese dos dados, uma matriz de síntese descrita em uma planilha de Excel ${ }^{\circ}$. Foram coletados dados como: periódico; país e ano de publicação; autor(es); título; desenho do estudo; principais resultados, fatores relacionados à qualidade da atenção e nível de evidência. Intentou-se com o instrumento, além de formar um banco de dados, mapear pontos pertinentes, integrar dados e caracterizar a amostra revisada. Desse modo, parte destes dados estão representados no Quadro 2.

\section{Quadro 2. Amostra final de artigos. Brasilia, Distrito Federal, 2020.}

\begin{tabular}{|c|c|c|c|c|c|c|}
\hline ESTUDO & PERIÓDICO & AUTOR(ES) & ANO & TÍTULO & $\begin{array}{l}\text { DESENHO DO } \\
\text { ESTUDO }\end{array}$ & $\begin{array}{l}\text { NIIVEL DE } \\
\text { EVIDÊNCIA }\end{array}$ \\
\hline E1 & $\begin{array}{l}\text { Revista de } \\
\text { Enfermagem } \\
\text { e Atenção à } \\
\text { Saúde }\end{array}$ & $\begin{array}{l}\text { Henz GS, Medeiros } \\
\text { CRG, Salvadori M }\end{array}$ & 2017 & A inclusão paterna durante pré-natal & Qualitativo & IV \\
\hline E2 & $\begin{array}{l}\text { Revista Online } \\
\text { de Pesquisa } \\
\text { Cuidado é } \\
\text { Fundamental }\end{array}$ & $\begin{array}{l}\text { Cardoso VEPS, Silva } \\
\text { Junior AJ, Bonatti } \\
\text { AF, Santos GWS, } \\
\text { Ribeiro TAN }\end{array}$ & 2018 & $\begin{array}{l}\text { A participação do parceiro na rotina pré- } \\
\text { natal sob a perspectiva da mulher gestante }\end{array}$ & Qualitativo & IV \\
\hline E3 & $\begin{array}{c}\text { Revista de } \\
\text { Enfermagem } \\
\text { do Centro- } \\
\text { Oeste Mineiro }\end{array}$ & $\begin{array}{l}\text { Caldeira LA, Ayres } \\
\text { LFA, Oliveira LVA, } \\
\text { Henriques BV }\end{array}$ & 2017 & $\begin{array}{l}\text { A visão da gestante acerca da participação } \\
\text { do homem no processo gestacional }\end{array}$ & Qualitativo & IV \\
\hline E4 & $\begin{array}{l}\text { BMC } \\
\text { Pregnancy and } \\
\text { childbirth }\end{array}$ & $\begin{array}{l}\text { Firouzan V, Noroozi } \\
\text { M, Farajzadegan Z, } \\
\text { Mirghafourvand M }\end{array}$ & 2019 & $\begin{array}{l}\text { Barriers to men's participation in perinatal } \\
\text { care: a qualitative study in iran }\end{array}$ & Qualitativo & IV \\
\hline E5 & $\begin{array}{c}\text { Texto e } \\
\text { Contexto } \\
\text { Enfermagem }\end{array}$ & $\begin{array}{l}\text { Holanda SM, Castro } \\
\text { RCMB, Aquin PS, } \\
\text { Pinheiro AKB, Lopes } \\
\text { LG, Martins ES }\end{array}$ & 2018 & $\begin{array}{c}\text { Influência da participação do companheiro } \\
\text { no pré-natal: satisfação de primíparas } \\
\text { quanto ao apoio no parto }\end{array}$ & Quantitativo & IV \\
\hline
\end{tabular}




\begin{tabular}{|c|c|c|c|c|c|c|}
\hline E6 & $\begin{array}{c}\text { Revista } \\
\text { Paulista de } \\
\text { Enfermagem }\end{array}$ & $\begin{array}{c}\text { Cavalcanti MAA, } \\
\text { Tsunechiro MA }\end{array}$ & 2018 & $\begin{array}{c}\text { O comportamento paterno na consulta } \\
\text { pré-natal }\end{array}$ & Qualitativo & IV \\
\hline $\begin{array}{c}\text { Eevista Online } \\
\text { de Pesquisa } \\
\text { Cuidado é } \\
\text { Fundamental }\end{array}$ & $\begin{array}{c}\text { Mello MG, Parauta } \\
\text { TC, Saldanha BL, } \\
\text { Lemos A }\end{array}$ & 2020 & $\begin{array}{c}\text { Participação do pai jovem no } \\
\text { acompanhamento do pré-natal: a visão do } \\
\text { profissional de saúde }\end{array}$ & Qualitativo & IV \\
\hline E8 & PLOS ONE & $\begin{array}{c}\text { Albuja AF, Sanchez } \\
\text { DT, Lee SJ, Lee JY, } \\
\text { Yadava S }\end{array}$ & 2019 & $\begin{array}{c}\text { The effect of paternal cues in prenatal care } \\
\text { settings on men's involvement intentions }\end{array}$ & Quantitativo & IV \\
\hline Saúde Coletiva & $\begin{array}{c}\text { Gomes R, Albernaz } \\
\text { L, Ribeiro CRS, } \\
\text { Moreira MCN, } \\
\text { Nascimento M }\end{array}$ & 2015 & $\begin{array}{c}\text { Linhas de cuidados masculinos voltados } \\
\text { para a saúde sexual, a reprodução e a } \\
\text { paternidade }\end{array}$ & $\begin{array}{c}\text { Opinião de } \\
\text { Especialista }\end{array}$ & VI \\
\hline E10 & $\begin{array}{c}\text { Enferm. Foco } \\
\text { Cavalcanti TRL, } \\
\text { Holanda VR }\end{array}$ & 2019 & $\begin{array}{c}\text { Participação paterna no ciclo gravídico- } \\
\text { puerperal e seus efeitos sobre a saúde da } \\
\text { mulher }\end{array}$ & $\begin{array}{c}\text { Revisão } \\
\text { Integrativa }\end{array}$ & VI \\
\hline Elaboração: Vieira AF, Aguiar RS, 2020.
\end{tabular}

A maior parte das publicações refere-se ao ano de 2018 e 2019 , com três publicações em cada ano (30\% em cada ano), seguidas do ano 2017 com dois (20\%), 2015 com um artigo (10\%) e 2020 também com um artigo (10\%). O desenho qualitativo foi o que mais prevaleceu dentre as pesquisas (seis artigos - 60\%). Sobre o nível de evidência dos artigos, houve maior prevalência de estudos não experimentais, como a pesquisa descritiva, correlacional e comparativa, com abordagem qualitativa e estudos de caso (80\%).

\section{DISCUSSÃO}

A análise desta temática dos resultados dos artigos permitiu a organização em três categorias temáticas principais: 1) Participação paterna no pré-natal e benefícios para a mulher; e 2) Oportunidades de melhorias para a participação paterna.

\section{Participação paterna no pré-natal e benefícios para a mulher}

No momento atual, percebe-se mudanças no que diz respeito à participação do homem durante o período gestacional em que se observa que este está participando mais ativamente da gravidez. Diante disso, incluir o homem nos atendimentos de pré-natal é uma forma capaz de proporcionar maior interesse na gestação e estimular que

\section{Diante disso,}

incluir o homem

nos atendimentos

de pré-natal é

uma forma capaz

de proporcionar

maior interesse na

gestação e estimular

que ele passe a

assumir maiores

responsabilidades

de cuidado com a

mulher e o recém-

nascido. ele passe a assumir maiores responsabilidades de cuidado com a mulher e o recém-nascido. ${ }^{17}$

É essencial que o homem proporcione à sua companheira apoio emocional para que esta se sinta mais segura durante a gestação. ${ }^{17-18}$ Ademais, ter o parceiro durante o atendimento de pré-natal auxilia na resolução das dúvidas que surgem sobre o ciclo gravídico e este ainda pode ser um apoio para relembrar as prescrições realizadas pelos profissionais de saúde. ${ }^{18}$

Quando as gestantes são perguntadas sobre a participação do companheiro durante a gravidez, as expressões de sentimentos usadas pela maioria das mulheres foram segurança, força e alegria, dentre outros sentimentos. ${ }^{19}$ Contudo, os homens mencionaram que dentre os motivos para não participarem das consultas de pré-natal está a falta de requerimento das mulheres e o fato delas serem acompanhadas por outros membros familiares durante as consultas. ${ }^{20}$

Ademais, outros fatores foram apresentados pelos homens para não participarem dos atendimentos, como a falta de consciência deles sobre a importância de sua participação no período do pré-natal e seu papel na saúde do recém-nascido, a ausência de conhecimento sobre a sua participação na gravidez, parto e cuidados pós-parto e a inexistência de uma interação apropriada entre casais devido às dificuldades de comunicação. ${ }^{20}$ 
A prática de seis ou mais consultas de pré-natal estiveram associadas à presença do parceiro como acompanhante da mulher. ${ }^{21}$ Além disso, o homem relata alegria com a gravidez em andamento e que na sua vivência de estar presente nos atendimentos de pré-natal possibilitou acompanhar e compreender melhor a gravidez não somente em relação aos cuidados com a mulher, mas também com os sentimentos de obrigação e decisões que devem ser tomadas em conjunto. ${ }^{22}$

O envolvimento paterno proporciona maior segurança em seu potencial de ser um bom pai e maiores intenções relatadas de aprender sobre a gravidez e se envolver em uma conduta positiva junto com sua parceira. ${ }^{23-24}$ Assim, no decorrer das consultas do pré-natal, manter o diálogo com o homem e a mulher são fundamentais para evitar possíveis estressores relacionados à gravidez. ${ }^{25}$

O acompanhamento paterno no decorrer do pré-natal apresenta melhoria no vínculo familiar, pois acaba propiciando uma maior aproximação entre o casal com demonstrações de afeto, aconchego e cumplicidade, diminuindo as divergências entre o casal. ${ }^{14} \mathrm{~A}$ experiência de estar presente durante este ciclo proporciona ao homem descobrir sentimentos afetuosos e amorosos capazes de construir vínculo entre pai-mãe-filho que possibilita uma união familiar. ${ }^{18}$

Quando o homem interage de forma permanente durante a gestação, o vínculo estabelecido entre mãe-feto aumenta e faz com que as transformações gravídicas sejam suportadas com maior clareza e aceitação, além de propiciar um ambiente mais seguro e acolhedor que possibilita a gestante realizar maior interação com o bebê e, assim, diminuir a ansiedade e preocupações. ${ }^{19}$

A presença do homem nas consultas de pré-natal, influencia na avaliação da mulher sobre o apoio físico e psicológico, além do acolhimenhto. ${ }^{21-22}$ Assim, o cuidado com a saúde da mulher vem revelando que a relação com o homem influencia profundamente o bem-estar da mulher na gestação e após o nascimento do filho, seja pela sua presença, aceitação e prazer de estar juntos. ${ }^{22}$ Constatação de que a paternidade afetiva tem um impacto notável no desenvolvimento físico, emocional e social dos filhos trazendo vantagens para a família e a sociedade como um todo, além de fazer toda a diferença para a mulher na questão da estabilidade física e emocional..$^{23}$

\section{Quando o homem} interage de forma permanente durante a gestação, o vínculo estabelecido entre mãe-feto aumenta e faz com que as transformações gravídicas sejam suportadas com maior clareza e aceitação, além de propiciar um ambiente mais seguro e acolhedor que possibilita a gestante realizar maior interação com o bebê e, assim, diminuir a ansiedade $e$ preocupaçóes.
Oportunidades de melhorias para a participação paterna

Para maior adesão paterna ao pré-natal, é fundamental que os profissionais de saúde criem oportunidades para que seja ofertado orientações e exames preventivos aos homens. Além disso, recomenda-se que a assistência seja realizada juntamente com as mulheres que estejam realizando o pré-natal. ${ }^{17}$

O Ministério da Saúde tem realizado estratégias para incluir o homem nas práticas de cuidado do ciclo gravídico-puerperal a partir da Rede Cegonha e da Lei do Acompanhante. Todavia, os serviços de saúde ainda não possibilitam espaços adequados para que haja envoltura e estímulo dos profissionais de saúde para que estas gestantes tragam consigo o cônjuge para as consultas de pré-natal. ${ }^{18}$

É necessário compreender que a atenção no pré-natal seja centrada na família, dispensando cuidados não apenas à mulher e ao feto, mas ao casal. No conceito de paternidade ativa, o homem se insere nesse contexto e é essencial sua participação no pré-natal para uma maior interação pai-filho. ${ }^{19}$

Acredita-se que a mídia tem um papel enfraquecido na criação da cultura dos homens na participação do pré-natal por julgar a gravidez e o parto como assunto privado. ${ }^{20}$ Contudo, é papel do enfermeiro orientar e estimular as gestantes quanto à presença do homem, não só na sala de parto, mas também no pré-natal, pois ele estará mais capacitado para proporcionar maior apoio à mulher e à criança desde o início do ciclo gravídico-puerperal. ${ }^{21}$

Os profissionais de saúde são capazes de trazer discussões amplas em relação à paternidade nas práticas educativas voltadas para gestantes e homens. A expectativa de que seja dada a importância necessária de intervenção dos profissionais, procurando, novas práticas voltadas à inserção dos pais adolescentes nos serviços de saúde. ${ }^{23}$

Assim, o reconhecimento e encorajamento à participação dos homens de distintos perfis em todas as etapas da gravidez (pré-natal, parto e pós-parto), respeitando o direito de escolha do acompanhante pela a mãe, é de fundamental importância e deve ser incentivado cada vez mais pelos profissionais de saúde..$^{25-26}$ 


\section{CONCLUSÃO}

A partir dos dados mencionados na revisão, pode-se observar que a participação paterna durante o pré-natal é de suma importância para favorecer maior vínculo entre o casal e proporcionar um ambiente acolhedor para o recém-nascido. Além disso, o suporte paterno durante o pré-na- tal é capaz de trazer sentimentos positivos para a mulher e isso influencia no decorrer da gestação. Desafios precisam ser superados para maior participação dos homens no acompanhamento pré-natal como dos horários das consultas e coincidir com o horário de trabalho, entre outras.

Diante destes fatos, é possível identificar a necessidade do acompanhamen- to paterno durante todo o pré-natal. O apoio paterno traz grandes benefícios em relação à saúde do recém-nascido e da gestante, sendo notório esta identificação de acordo com os artigos selecionados para este estudo.

Conclui-se, portanto, que a participação do pai gera um vínculo muito importante tanto na vida da criança como na da gestante.

\section{REFERÊNCIAS}

1. Camillo BS, Nietsche EA, Salbego C, Cassenote LG, Dal Osto DS, Böck A. Ações de educação em saúde na Atenção Primária à gestantes e puérperas: revisão integrativa. Rev enferm UFPE, 2016;10(Supl. 6):4894-901.

2. Ministério da Saúde (BR). Guia do Pré-Natal do Parceiro para Profissionais de Saúde. Rio de Janeiro: Ministério da Saúde; 2015.

3. Lima CS, Aguiar RS. Acesso dos homens aos serviços de atenção primária à saúde: uma revisão integrativa. Research, Society and Development. 2020;9(4):e157943027.

4. Pereira J, Klein C, Meyer DE. PNAISH: uma análise de sua dimensão educativa da perspectiva de gênero. Saúde Soc. 2019;28(2):132-146.

5. Oliveira VB, Aguiar RS. Conhecimento da política de saúde do homem e a relação com a atenção à saúde. Saúde Coletiva (Barueri). 2020;10(55):2985-3002.

6. Balica LO, Aguiar RS. Percepções paternas no acompanhamento pré-natal. Rev Aten Saúde. 2019;17(61):114-26.

7. Fabro MRC, Lima MP. A experiência paterna de pais de "primeira viagem" no cuidado do bebê nos primeiros três meses de vida. Atas CIAIQ. 2017;2(1):166-75.

8. Aguiar RS, Santana DC, Santana PC. A percepção do enfermeiro da estratégia saúde da família sobre a saúde do homem. Rev Enferm Cent-Oest Min. 2015;5(3):1844-1854.

9. Miranda SVC, Oliveira PSD, Moraes VCM, Vasconcelos LCF. Necessidades e reivindicações de homens trabalhadores rurais frente à atenção primária à saúde. Trab Educ Saúde. 2019;18(1):e0022858.

10. Silva AN, Silva AS, Silva ARV, Araújo TME, Rebolças CBA, Nogueira LT. A avaliação da atenção primária à saúde na perspectiva da população masculina. Rev Bras Enferm. 2018;71(2):255-63.

11. Oliveira JPF, Barbosa RVF, Gottems LBD. A participação e as expectativas do homem na gestação e a sua adesão no pré-natal: em busca de evidências. Brasília: UCB; 2012.

12. Lima RC, Aguiar RS. Experiência paterna com o recém-nascido a partir das orientações de enfermagem. Rev Cereus. 2020;12(1):193-202.

13. Pluye P, Hong QN. Combining the power of stories and the power of numbers: mixed methods research and mixed studies reviews. Annu Rev Public Health. 2014;35(1):29-45.

14. Donato $H$, Donato $M$. Etapas na condução de uma revisão sistemática. Acta Med Port. 2019;32(3):227-235.

15. OCEBM Levels of Evidence Working Group. The Oxford 2011 Levels of Evidence. Oxford: Oxford Centre for Evidence-Based Medicine; 2011.

16. Braun V, Clarke V. Using thematic analysis in psychology. Qual Res Psychol. 2006;3(2):77-101.

17. Henz GS, Medeiros CRG, Salvadori M. A inclusão paterna durante pré-natal. Rev Enferm Atenção Saúde. 2017;6(1):52-66.

18. Cardoso VEPS, Silva Junior AJ, Bonatti AF, Santos GWS, Ribeiro TAN. A participação do parceiro na rotina pré-natal sob a perspectiva da mulher gestante. J Res Fundam Care. 2018; 10(3):856-62.

19. Caldeira LA, Ayres LFA, Oliveira LVA, Henriques BV. A visão da gestante acerca da participação do homem no processo gestacional. Rev Enferm Cent-Oest Min. 2017;7:e1417.

20. Firouzan V, Noroozi M, Farajzadegan Z, Mirghafourvand M. Barriers to men's participation in perinatal care: a qualitative study in iran. BMC Pregnancy and childbirth. 2019;19:45.

21. Holanda SM, Castro RCMB, Aquin PS, Pinheiro AKB, Lopes LG, Martins ES. Influência da participação do companheiro no pré-natal: satisfação de primíparas quanto ao apoio no parto. Texto Contexto Enferm. 2018;27(2):e3800016.

22. Cavalcanti MAA, Tsunechiro MA. O comportamento paterno na consulta pré-natal. Rev Paul Enferm. 2018;29(1):39-46.

23. Mello MG, Parauta TC, Saldanha BL, Lemos A. Participação do pai jovem no acompanhamento do pré-natal: a visão do profissional de saúde. J Res Fundam Care. 2020;12:95-100.

24. Albuja AF, Sanchez DT, Lee SJ, Lee JY, Yadava S. The effect of paternal cues in prenatal care settings on men's involvement intentions. PLOS One. 2019;14(5):e0216454.

25. Gomes R, Albernaz L, Ribeiro CRS, Moreira MCN, Nascimento M. Linhas de cuidados masculinos voltados para a saúde sexual, a reprodução e a paternidade. Cienc Saúde Colet. 2015;21(5):1545-52.

26. Cavalcanti TRL, Holanda VR. Participação paterna no ciclo gravídico-puerperal e seus efeitos sobre a saúde da mulher. Enferm Foco. 2019;10(1):93-98 